\title{
Genèse du \\ processus addictif
}

Hélène Scarna

> L'addiction se développe chez des sujets vulnérables à la suite d'une consommation répétée de drogues. La recherche de l'origine de cette vulnérabilité que nous avons réalisée au cours d'entretiens psychologiques avec 312 patients atteints de troubles addictifs dans un centre de soin, d'accompagnement et de prévention en addictologie, nous conduit à proposer une hypothèse quant à la genèse du processus addictif. Cette hypothèse prend ses racines dans l'articulation entre théories psychodynamiques et neurobiologiques du développement des affects. L'observation clinique révèle la fréquence des souffrances narcissiques chez ces patients. C'est donc dans la constitution historique du narcissisme que les origines du comportement addictif ont été recherchées. <

\section{Développement du narcissisme biopsychique}

Le psychanalyste Bela Grunberger définit un noyau narcissique primitif qui est la trace de la cénesthésie ${ }^{1}$ fœtale [1]. Il s'agit d'une expérience organique qui laisserait son empreinte chez tout individu, durant toute sa vie. L'état élationnel ${ }^{2}$ prénatal serait la source de la nostalgie de l'être humain pour son séjour prénatal. Pour expliquer que l'état cénesthésique prénatal se révèle avoir une valence positive, être source de complétude, il faut formuler l'hypothèse que le cerveau du fœtus est capable d'associer à cet état une valeur attractive. Des études ont montré que l'activation des récepteurs $\mu$ des opiacés était à l'origine de la coloration plaisante non consciente d'une sensation [2]. Le système opioïde endogène semble donc central dans l'attribution d'une valence agréable à une percep-

Vignette (๔ Inserm/Éric Dehausse/Yasmina Saoudi).

1 La cénesthésie est une perception vague que nous avons de notre être ou de notre corps, indépendamment du concours des sens (e.g., fatigue, bien-être), ou grâce à la sensibilité organique faiblement consciente qui provient de tous nos organes et tissus, y compris les organes des sens (e.g., faim, excitation sexuelle).

2 État de bonheur complet...

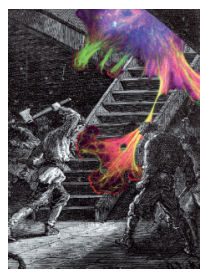

tion. Une étude réalisée sur des souriceaux privés du gène codant le récepteur $\mu$ des opiacés a par ailleurs montré l'existence d'un déficit dans leur comportement d'attachement à leur mère [3]. L'attribution d'une valence positive à l'expérience prénatale semble donc nécessaire au développement du processus d'attachement de l'enfant à sa mère. Chez l'homme, le système opioïde apparaît dès la $12^{\mathrm{e}}$ semaine de vie intra-utérine, ce qui rend plausible sa mise en jeu dans l'attribution d'une valence positive à la sensorialité prénatale. Les données concernant le toucher « affectif » sont en faveur de cette hypothèse [4]. En effet, la douceur d'un toucher active le système opioïde endogène. Ksenia Bystrova, pédiatre russe, propose que les mouvements du fœtus dans le liquide amniotique produisent des oscillations du duvet couvrant son corps, susceptibles de stimuler les fibres sensibles au toucher léger. Ce massage prénatal aurait ainsi un effet plaisant et apaisant, et favoriserait la croissance fœtale [5].

En ce qui concerne la période post-natale, le pédiatre et psychanalyste britannique Donald Winnicott souligne le rôle majeur de l'environnement dans le développement du narcissisme de l'enfant, qui conduit à la constitution du self. II parle de "préoccupation maternelle primaire » [6] pour désigner un état d'hypersensibilité de la mère aux tous premiers besoins du nourrisson. $0 r$, l'ocytocine joue un rôle important en début de vie, car ce neuropeptide favorise cette attention préférentielle et l'attractivité mutuelle entre la mère et le bébé, et 
donc la mise en place des processus d'attachement sécurisés. II a ainsi été montré, chez la souris, que l'ocytocine permettait une sensibilisation du cortex auditif des mères aux appels de détresse de leurs petits lorsqu'ils étaient loin du nid. En modulant la plasticité corticale, l'ocytocine augmente la saillance perceptive des stimulus acoustiques sociaux chez la mère, ce qui induit son comportement de récupération de ses petits pour les ramener dans son nid [7]. Un tel comportement fait écho au concept de Donald Winnicott de «préoccupation maternelle primaire », définie comme l'identification de la mère avec son enfant, lui permettant de savoir ce que l'enfant ressent et d'être en mesure de fournir presque exactement ce dont l'enfant a besoin [6].

\section{Vulnérabilité narcissique liée aux traumas précoces}

Les traumas de l'enfance peuvent avoir différentes origines: la négligence ou la maltraitance, mais aussi des relations précoces inadaptées passant souvent inaperçues et ne laissant aucune trace dans la mémoire explicite. Nous nous intéressons à ce dernier cas.

Donald Winnicott considère qu'au-delà de la survenue d'un évènement traumatique temporellement circonscrit, c'est le mode inadéquat de réponse ordinaire de l'environnement aux besoins du Moi du bébé qui peut être traumatisant. Lorsque l'adaptation de la mère n'est pas suffisante, quand elle est inapte à ressentir les besoins du nourrisson, elle ne répond pas à son geste spontané et y substitue le sien propre, ce qui conduit à la soumission du nourrisson. Cet empiètement produit le développement d'un faux self chez l'enfant, qui engendre un sentiment d'inanité, et à partir duquel il élabore un ensemble de relations artificielles [8]. Le faux self a une fonction défensive : il dissimule le vrai self et évite son anéantissement en se soumettant aux exigences de l'environnement. Ces traumas narcissiques ne relèvent pas de la frustration, mais d'une perte de la capacité d'être ou de se sentir être, et rendent le narcissisme vulnérable.

Dans le champ de la biologie, le trauma a été défini à partir du concept de stress. Il faut souligner que plus l'individu confronté à un stress est jeune, moins il est apte à l'anticiper et à le contrôler par une action physique ou psychique adaptée, ce qui augmente sa sensibilité. L'impact cérébral des traumas précoces persiste jusqu'à l'âge adulte. Ses conséquences se traduisent par une plus grande sensibilité au stress et un risque accru de développer certaines maladies psychiques ou somatiques. Ainsi, une vulnérabilité de l'adulte à diverses maladies pourrait être biologiquement «programmée » pendant la période fœtale. Ce constat a ensuite été étendu à la période postnatale précoce et a donné naissance au concept de «programmation périnatale», qui tient compte de l'influence des facteurs environnementaux dans le développement des systèmes biologiques [9] $(\rightarrow)$; ses mécanismes sont de nature épigénétique et concernent, entre autres, $\rightarrow$ Voir la Synthèse de M. Rincel et al., $\mathrm{m} / \mathrm{s} \mathrm{n}^{\circ} 1$, janvier 2016, page 93 les récepteurs de l'ocytocine [10]. Par exemple, chez le rat, un stress se produit lorsque le niveau de soins maternels (léchage/toilettage) est faible. Moins les ratons ont de contacts avec la mère au cours de la première semaine postnatale, plus leurs réactivités comportementale et neuroendocrinienne au stress sont fortes. En revanche, si ces ratons nouveau-nés sont adoptés par une mère plus maternante, ils ont une réactivité normale au stress, ce qui montre qu'il existe une relation directe entre la qualité des soins maternels et le développement phénotypique de la progéniture.

\section{Hypothèse sur la genèse du processus addictif [11]}

Sachant que les drogues en général, et l'héroïne en particulier ${ }^{3}$, activent le système opioïde endogène, ces produits exogènes viendraient actualiser les traces cénesthésiques formées au cours de la vie prénatale et répliqueraient ainsi l'état de complétude narcissique vécue par le sujet avant la naissance. En effet, l'activation du système opioïde lui permet, au moins lors des premières consommations de la drogue, de surmonter les besoins vitaux, de soulager toute souffrance grâce à la fonction analgésique des opiacés, et de retrouver un état de bien-être proche de celui qu'il a vécu in utero. C'est, par exemple, la plénitude émotionnelle procurée par l'héroïne avec l'impression d'être distancié de l'environnement, protégé de tout excès d'excitation et de l'empiètement psychique par l'entourage. Sur la base de ce postulat, une hypothèse mettant en jeu la vie postnatale, pour rendre compte du risque addictif chez les personnalités narcissiquement vulnérables, peut être avancée. Nous proposons que l'impact psychique de la première expérience avec la drogue est d'autant plus marqué que les expériences postnatales de complétude narcissique ont été rares. Si l'expérience prénatale initiale n'a pas pu être répétée après la naissance, la drogue réactualisera cette trace fœtale et donnera une impression de révélation au sujet expérimentateur de la drogue. Au contraire, la variété et la qualité des interactions précoces pendant la période postnatale constitueraient une protection contre le risque addictif. $\varepsilon n$ effet, dans cette première étape de développement postnatal, chaque expérience de satisfaction non seulement réactualise l'état de complétude vécu in utero, mais aussi oriente la pulsion de l'enfant du côté de l'accomplissement par lui-même de ces expériences de plaisir (autoérotisme).

Pour finir, les concepts winnicottiens de vrai et faux self [8] permettent un pas de plus dans la compréhension de la fonction désinhibitrice des drogues, qui correspond à une levée des défenses que constitue le faux self. En effet, au début de la vie, le vécu de complétude narcissique permet l'émergence du geste spontané issu

3 L'héroïne active directement les récepteurs des opiacés, mais toutes les drogues, y compris l'alcool, les activent indirectement en provoquant la libération des peptides opioïdes endogènes. 
du vrai self, dont le destin dépendra de la façon dont il est accueilli par l'environnement de l'enfant. Ainsi, si le vrai self ne peut se développer en raison d'une adaptation insuffisante de l'environnement aux pulsions du nourrisson, il sera masqué par la production d'un faux self. Dans cette conjoncture, nous avancerons l'hypothèse que pour le consommateur de drogue, elle est une façon d'entrer en contact avec son vrai self et, s'il n'a préalablement trouvé aucun autre moyen pour y parvenir, la répétition de cette expérience deviendra compulsive afin de maintenir vivant le sentiment d'existence.

L'écoute d'un patient sous traitement de substitution aux opiacés éclaire cette hypothèse. II évoque ses prises matinales d'héroïne qui lui permettaient de se lever, d'aller travailler et de supporter des tâches professionnelles ennuyeuses et répétitives, mais aussi d'aller vers les autres avec aisance et bonne humeur. II met donc en avant, non plus le côté orgasmique du plaisir revendiqué en premier lieu, mais plutôt l'effet «normalisant » du bien-être procuré par la drogue. Lorsqu'on lui demande s'il se sent être plus lui-même avec ou sans héroïne, il répond que c'est l'héroïne qui lui permet d'être vraiment lui-même, d'exprimer ce qu'il est réellement. Une des fonctions de la consommation de drogue serait donc de lever les mécanismes de défense rigides qui contraignent le self. $\diamond$

Genesis of the addictive process

\section{LIENS D'INTÉRÊT}

L'auteure déclare n'avoir aucun lien d'intérêt concernant les données publiées dans cet article.

\section{RÉFÉRENCES}

1. Grunberger B. Le narcissisme, essai de psychanalyse. Paris : Payot, 1993 : $350 \mathrm{p}$.

2. Berridge K, Robinson T, Aldridge J. Dissecting components of reward: 'liking', 'wanting', and learning. Curr Opin Pharmacol $2009 ; 9: 65-73$.

3. Moles A, Kieffer B, D'amato F. Deficit in attachment behavior in mice lacking the mu-opioid receptor gene. Science 2004 ; 304 : 1983-6.

4. Mcglone F, Wessberg J, Olausson $\mathrm{H}$. Discriminative and affective touch: sensing and feeling. Neuron $2014 ; 82: 737-55$.

5. Bystrova K. Novel mechanism of human fetal growth regulation: a potential role of lanugo, vernix caseosa and a second tactile system of unmyelinated low-threshold C-afferents. Medical Hypoth 2009 ; 72 : 143-6.

6. Winnicott $\mathrm{D}$. La préoccupation maternelle primaire. In : De la pédiatrie à la psychanalyse. Paris : Payot, 1992 (1956): 285-91.

7. Marlin B, Mitre M, D'amour J, et al. Oxytocin enables maternal behavior by balancing cortical inhibition. Nature $2015 ; 520: 499-504$.

8. Winnicott D. Distorsion du moi en fonction du vrai et du faux « Self ». In : Processus de maturation chez l'enfant. Développement affectif et environnement. Paris : Payot, 1970 (1960): 115-31.

9. Rincel M, Lépinay A, Gabory A, et al. Environnement précoce et vulnérabilité neuropsychiatrique. Med Sci (Paris) 2016 ; $32: 93-9$

10. Perkeybile AM, Carter CS, Wroblewski KL, et al. Early nurture epigenetically tunes the oxytocin receptor. Psychoneuroendocrinology $2019 ; 99: 128-36$.

11. Scarna H. Genesis of the heroin-induced addictive process: articulation between psychodynamic and neurobiological theories. Front Psychiatry $2020 ; 11: 524764$.
TIRÉS À PART

H. Scarna

$\mathrm{P}$ ossédées du Malin au Moyen-ÂAge, les sorcières hystériques sont vouées au bûcher. Enfermées au xvı ${ }^{e}$ siècle, maltraitées, elles rejoignent la Cour des Miracles de l'Hospice de la Vieillesse-Femmes à la Salpêtrière... Jusqu'à ce que le Dr JeanMartin Charcot (1825-1893) mène le combat qui transforme l'ancien hospice en hôpital : l'École de la Salpêtrière de

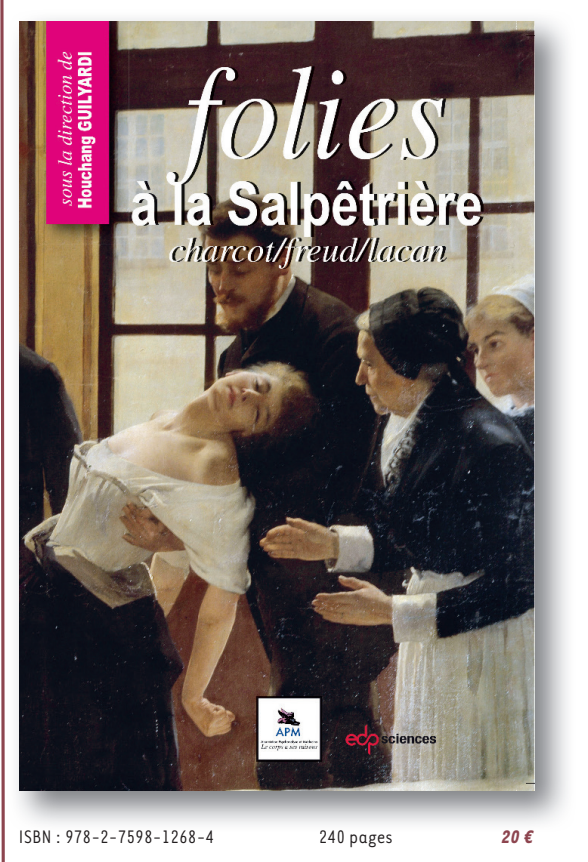
Paris est née, qui devient lieu de recherche, d'enseignement et de soins, de renommée internationale.

Jean Martin Charcot n'a pas bonne presse, et pourtant... Hystérie et folie traversent les siècles, prenant les formes de «l'air du temps ».

De l'utérus migrateur d'Hippocrate aux recherches neurologiques de Charcot. Du désir inconscient avec Freud à la jouissance du parlêtre chez Lacan... C'est à cette traversée historique et conceptuelle que nous convie cet ouvrage.

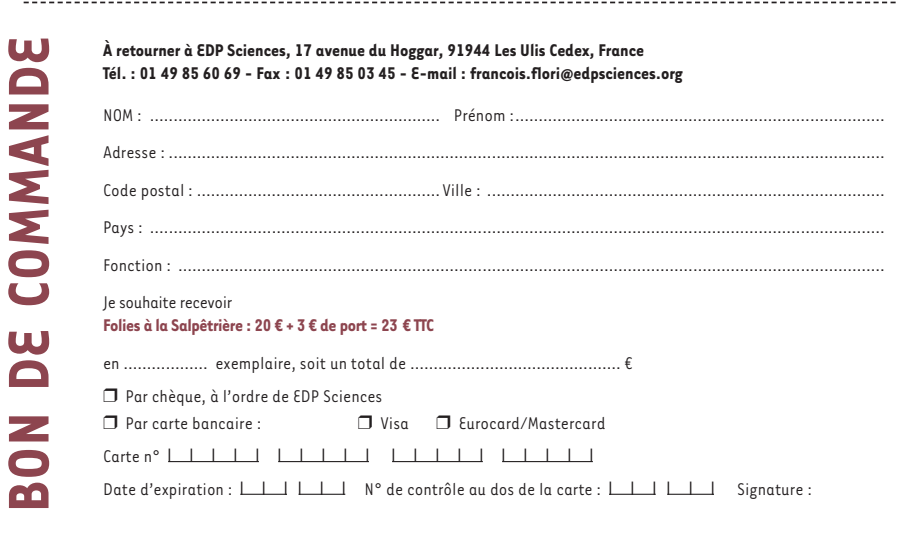

